\title{
LEGUMINOSAS DEL NORTE DEL ESTADO DE NUEVO LEÓN, MÉXICO
}

\author{
Eduardo Estrada CAstillón ${ }^{1}$, José Ángel Villarreal Quintanilla ${ }^{2}$ \\ Y ENRIQUE JURADO ${ }^{1}$
}

\begin{abstract}
${ }^{1}$ Facultad de Ciencias Forestales, Universidad Autónoma de Nuevo León Apdo. postal 41, 67700 Linares, N. L., México

${ }^{2}$ Departamento de Botánica, Universidad Autónoma Agraria Antonio Narro

Buenavista 25315 Saltillo, Coahuila
\end{abstract}

\section{RESUMEN}

Se estudió la diversidad de especies de leguminosas en el norte del estado de Nuevo León, contabilizando 98 taxa en total. Se registraron 38 géneros, 94 especies y 26 taxa infraespecíficos de este grupo de plantas; la familia Fabaceae comprende 18 géneros y 42 especies, Mimosaceae 10 y 30 y Caesalpiniaceae 10 y 22 respectivamente. Los géneros con mayor número de especies son Acacia (12), Dalea (10), Mimosa (6), Senna (5); Caesalpinia (4) y Bauhinia (4). Del total de 98 taxa, las leguminosas arbustivas y herbáceas son las más abundantes en la zona, representando $47 \%$ (46 especies) y 46\% (45 especies) respectivamente, las arbóreas representan 7\% (7). La mayor cantidad de taxa se registró en el matorral subinerme (52), matorral submontano (48) y bosque de encino-pino (44), mientras que los mezquitales albergan el menor número de especies (23).

Palabras clave: Diversidad, flora, leguminosas, México, Nuevo León.

\begin{abstract}
A study of the diversity of legumes in the northern part of the state of Nuevo León was undertaken. 38 genera, 94 species and 26 infraspecific taxa of legumes were recorded. The family Fabaceae comprises 18 genera and 42 species, Mimosaceae 10 and 30 and Caesalpiniaceae 10 and 22 respectivelly. The genera with highest number of species are Acacia (12), Dalea (10), Mimosa (6), Senna (5), Caesalpinia (4) and Bauhinia (4). Shrubby legumes are the most abundant in the zone, representing 47\% (46 species); the herbaceous ones constitute $46 \%$ (45) and the arboreal ones comprise 7\% (7). The highest number of taxa was recorded on semithorn shrubland (52) piedmont scrub (48), oak-pine forest (44), while the mezquite shrublands shelter the lowest number of species (23).
\end{abstract}

Key words: Diversity, flora, legumes, Mexico, Nuevo Leon. 


\section{INTRODUCCIÓN}

El grupo de las leguminosas está constituido por tres familias: Caesalpiniaceae, Mimosaceae y Fabaceae (Cronquist, 1988; Barneby, 1989). Se encuentra ampliamente diversificado y distribuido en todo el territorio nacional mostrando variadas formas biológicas. En varios estados del norte de México algunos taxa son abundantes sobre grandes extensiones, como Acacia rigidula, A. constricta, A. berlandieri y Prosopis glandulosa, asociados a diversas comunidades de matorral xerófilo (Estrada y Martínez, 2000). Algunas especies predominan en áreas con disturbio y constituyen un serio problema en campos de cultivo abandonados, donde su erradicación es difícil, destacando en este grupo Acacia farnesiana y Mimosa aculeaticarpa (Estrada y Martínez, op. cit.).

El norte del estado de Nuevo León está conformado por extensas planicies y serranías que no sobrepasan los $2500 \mathrm{~m}$ de altitud, la vegetación predominante es de tipo matorral, donde algunas leguminosas son los elementos preponderantes, como el "chaparro prieto" (Acacia rigidula), "tenaza" (Havardia pallens), "'huajillo" (Acacia berlandieri), "vara dulce" (Eysenhardtia texana), "huizache", (Acacia farnesiana), "uña de gato" (Acacia greggii), "palo verde" (Cercidium macrum), "retama" (Parkinsonia aculeata), "ébano" (Ebenopsis ebano), "gatuño" (Mimosa aculeaticarpa) y "mezquite" (Prosopis glandulosa).

Entre los diferentes usos que la población humana hace de las leguminosas destacan los de postería para cercas, material de construcción, enseres domésticos, mangos para hachas, azadones, sillas de montar, figuras decorativas de ébano, carbón y leña.

Debido al intenso aprovechamiento de los representantes de este grupo de plantas en la región septentrional de México, es de interés conocer la diversidad y distribución de las mismas en el estado de Nuevo León, específicamente en los municipios del norte de la entidad, en donde han sido poco estudiadas.

\section{DESCRIPCIÓN DEL ÁREA DE ESTUDIO}

El área de estudio comprende $23,867 \mathrm{~km}^{2}$, abarcando el territorio de los 20 municipios ubicados al norte del estado de Nuevo León: Abasolo, Agualeguas, Anáhuac, Bustamante, Cerralvo, Ciénega de Flores, El Carmen, General Treviño, Hidalgo, Higueras, Lampazos de Naranjo, Los Aldamas, Los Herreras, Melchor Ocampo, Mina, Parás, Sabinas Hidalgo, Salinas Victoria, Vallecillo y Villaldama (Fig. 1).

Fisiografía: La zona se caracteriza por extensas llanuras, lomeríos bajos y escasas montañas con intervalos altitudinales entre los 150 y los $1300 \mathrm{~m}$, con 
Estrada et al.: Leguminosas del norte de Nuevo León

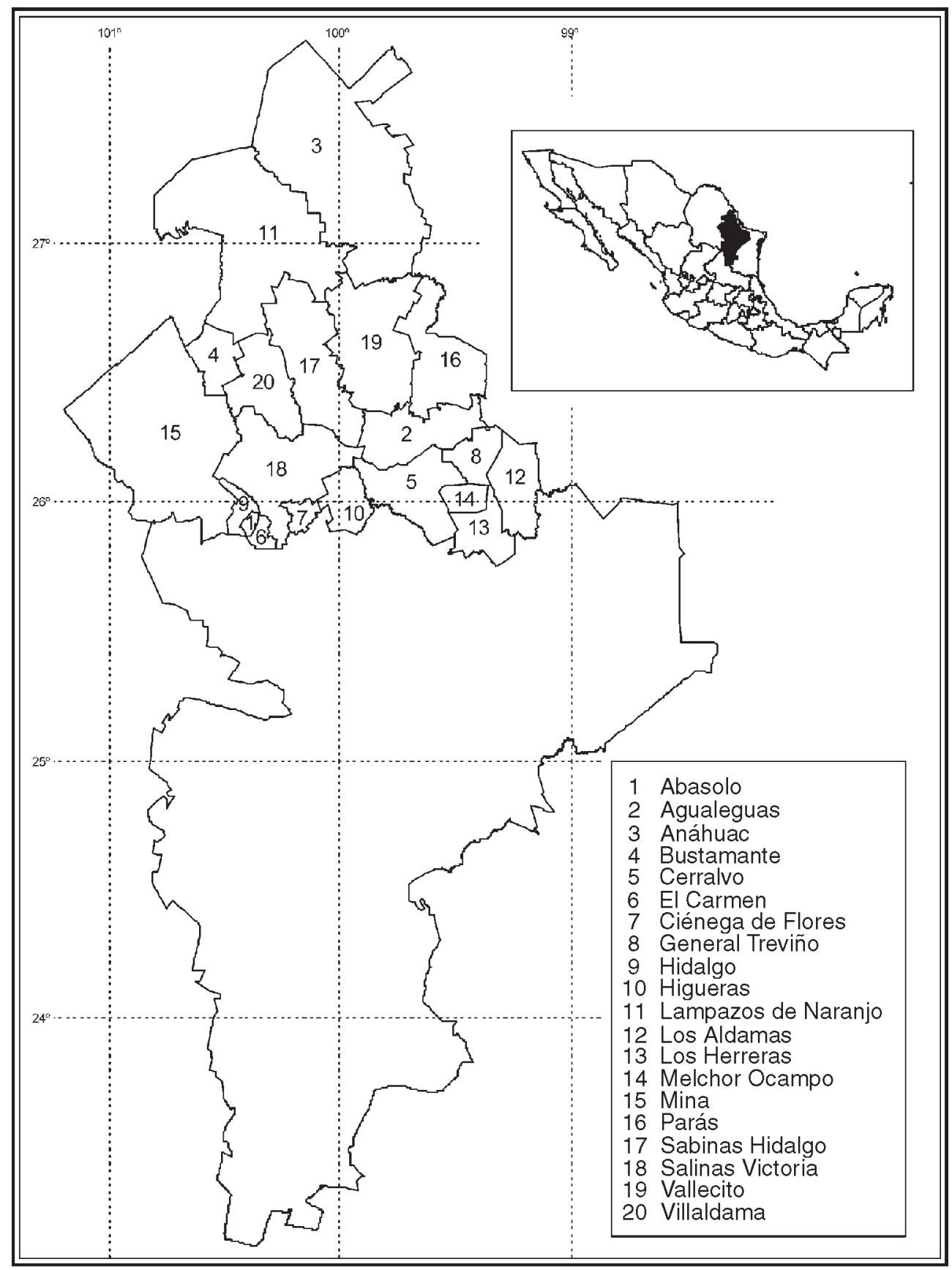

Fig. 1. Mapa del estado de Nuevo León mostrando los 20 municipios que comprende el área de estudio. 
afloramientos de rocas calcáreas pertenecientes al Cretácico Superior y conglomerados sobreyacentes del Terciario. Dentro de la región estudiada se localizan cadenas cerriles de tipo plegado con lomeríos donde predominan suelos de tipo litosol de origen residual y asociados a ellos se encuentran regosoles calcáricos. En los valles intermontanos prevalecen suelos salinos, yesosos o yermosoles gípsicos, xerosoles háplicos y cálcicos, limitados casi siempre por el caliche (Anónimo, 1981).

Clima: De acuerdo con García (1973), los climas predominantes en el extremo norte del estado de Nuevo León son de tipo seco y semiseco, la precipitación anual oscila entre 400 y $600 \mathrm{~mm}$ y la temperatura media anual es mayor de $22^{\circ} \mathrm{C}$. Para la región nororiental del estado predomina la variante semiseca muy cálida $\mathrm{BS}_{0} \mathrm{hx}^{\prime}(\mathrm{w})\left(\mathrm{e}^{\prime}\right)$ con lluvia escasa en todo el año. Este tipo de clima se presenta en los municipios de Sabinas Hidalgo, Lampazos de Naranjo, Vallecillo, Los Aldama, Higueras, parte de Cerralvo y parte de Villaldama.

El clima de tipo seco $\mathrm{BS}_{0}(\mathrm{~h}) \mathrm{hw}\left(\mathrm{e}^{\prime}\right)$ prevalece en la porción norte y noroeste, es muy seco semicálido con lluvia en verano. Con un porcentaje de lluvia invernal de 5-10.2, la precipitación anual total varía entre los 200 y los $400 \mathrm{~mm}$; la temperatura media anual es de 18 a $22^{\circ} \mathrm{C}$. Este clima se presenta en porciones de la región noroccidental del estado en los municipios de Mina, García, Doctor González, Ciénega de Flores, Salinas Victoria, Sabinas Hidalgo, Villaldama, Bustamante, San Nicolás Hidalgo y Abasolo (Anónimo, 1981). La única zona con clima semicálido moderadamente húmedo $(\mathrm{A}) \mathrm{C}\left(\mathrm{w}_{0}\right)$ en el área de estudio corresponde a la Sierra de Picachos (municipio Sabinas Hidalgo).

Vegetación: En el área de estudio se presentan cuatro comunidades vegetales principales: matorral submontano, mezquital, matorral subinerme y bosque de encino-pino.

El matorral submontano ocupa una superficie de aproximadamente $5,000 \mathrm{~km}^{2}$, cerca de $8 \%$ del territorio del estado de Nuevo León, y presenta su máxima distribución entre los 600 y los $800 \mathrm{~m}$ de altitud. Esta comunidad vegetal está conformada por elementos inermes y espinosos con alturas entre 2 y $4 \mathrm{~m}$ (White, 1940; Muller, 1939; Johnston, 1963; Miranda y Hernández X, 1963; RojasMendoza, 1965; Rzedowski, 1978). Los taxa predominantes son Helietta parvifolia, Forestiera angustifolia, Fraxinus greggii, Cordia boissieri, Gochnatia hypoleuca, Amyris madrensis, Amyris texana, Neopringlea integrifolia, Karwinskia humboldtiana, Malphigia glabra, Acacia berlandieri, Acacia rigidula, Acacia greggii, Celtis pallida, Randia rhagocarpa, Sideroxylon lanuginosum, Cercidium macrum, Zanthoxylum fagara, Leucophyllum frutescens y Havardia pallens.

El mezquital engloba comunidades dominadas por Prosopis glandulosa var. torreyana y $P$. glandulosa var. glandulosa que se distribuyen de manera irregular a lo largo y ancho del territorio estatal ocupando zonas que han sido perturbadas por cultivo y después abandonadas (Rojas-Mendoza, 1965; Anónimo, 1981). 
Frecuentemente asociados a esta comunidad vegetal se encuentran Forestiera angustifolia, Leucophyllum frutescens, Ziziphus obtusifolia, Acacia rigidula, A. berlandieri, Celtis pallida y Karwinskia humboldtiana.

El matorral subinerme está conformado por plantas arbustivas con alturas entre 1-1.5 m (Anónimo, 1981). Los elementos predominantes son Larrea tridentata, Flourensia cernua, Acacia neovernicosa, Leucophyllum texanum y Prosopis glandulosa. Entre las especies asociadas más comunes se encuentran Fouquieria splendens, Mimosa aculeaticarpa, Yucca filifera, Opuntia imbricata, O. leptocaulis, Condalia ericoides, Celtis pallida, Acacia greggii, A. roemeriana y Koeberlinia spinosa.

El bosque de encino-pino se localiza en las partes medias y más altas de los macizos montañosos, por encima de los $850 \mathrm{~m}$ de altitud; las especies más comunes son Quercus laceyi, Q. canbyi, Q. polymorpha y $Q$. rysophylla. Las coníferas más frecuentes son Pinus cembroides, Juniperus monosperma y J. deppeana.

\section{MÉTODOS}

Durante los años 2000-2001 se realizaron colectas de material botánico de leguminosas a lo largo y ancho de la superficie que comprende la porción norte del estado de Nuevo León. Los sitios de muestreo se establecieron en todas las comunidades vegetales reconocidas para el estado (Rojas-Mendoza, 1965; Anónimo, 1981). En cada lugar se anotó la altitud, pendiente, exposición, tipo de suelo, coordenadas geográficas, nombre de la localidad y municipio al que pertenece. Los ejemplares fueron herborizados, identificados e incluidos en la colección científica del herbario de la Facultad de Ciencias Forestales (CFNL) de la Universidad Autónoma de Nuevo León; juegos incompletos de ejemplares se depositaron en los herbarios ANSM, MEXU Y TEX. Se revisaron los herbarios TEX y UNL para completar el inventario de especies del área estudiada.

\section{RESULTADOS}

Se registró un total de 98 taxa, correspondientes a 38 géneros, 94 especies y 26 taxa infraespecíficos de leguminosas en el norte del estado de Nuevo León (ver Apéndice). De las tres familias que constituyen el orden Fabales, las Fabaceae comprenden 18 géneros y 42 especies, las Mimosaceae 10 y 30 y las Caesalpiniaceae 10 y 22 respectivamente (Fig. 2). Los géneros con mayor número de especies son Acacia (12), Dalea (10), Mimosa (6), Senna (5), Caesalpinia (4) y Bauhinia (4), mientras que otros seis registran tres especies cada uno (Fig. 3). 


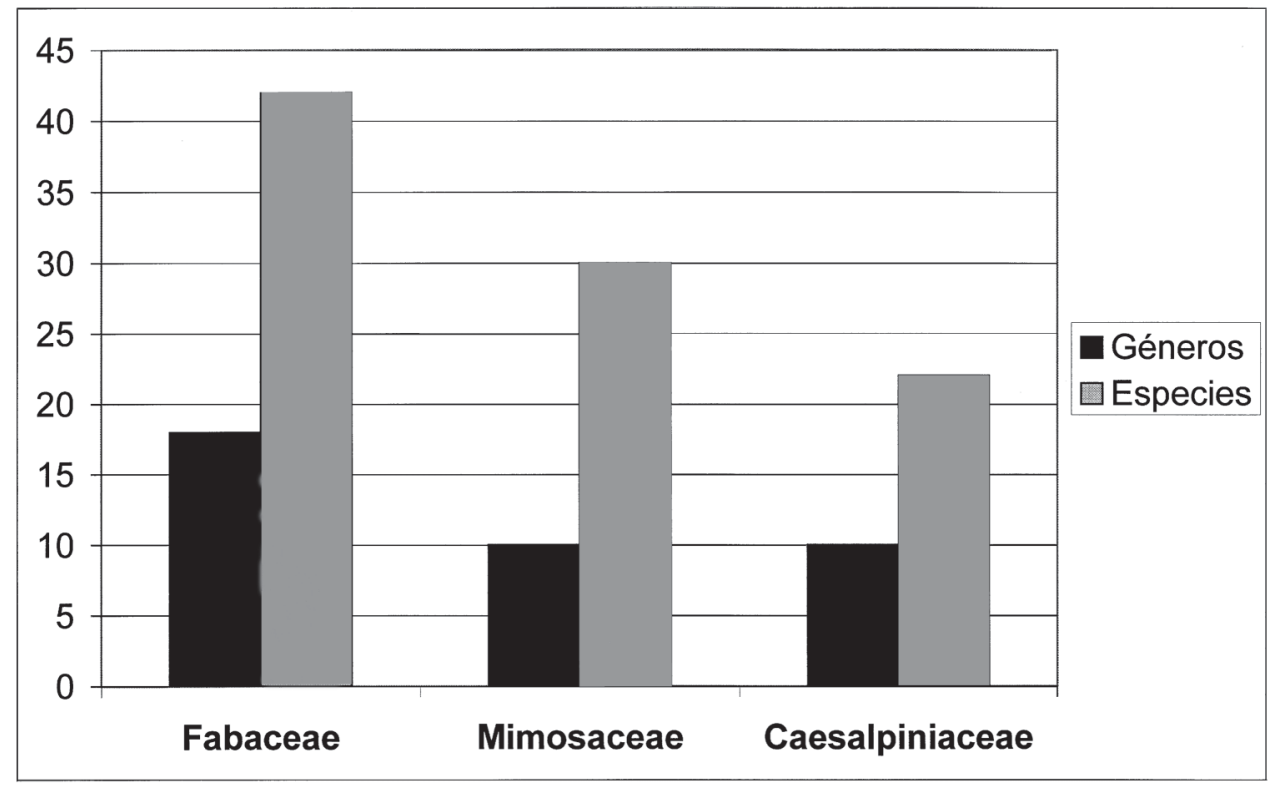

Fig. 2. Número de géneros y especies registrados por familia.

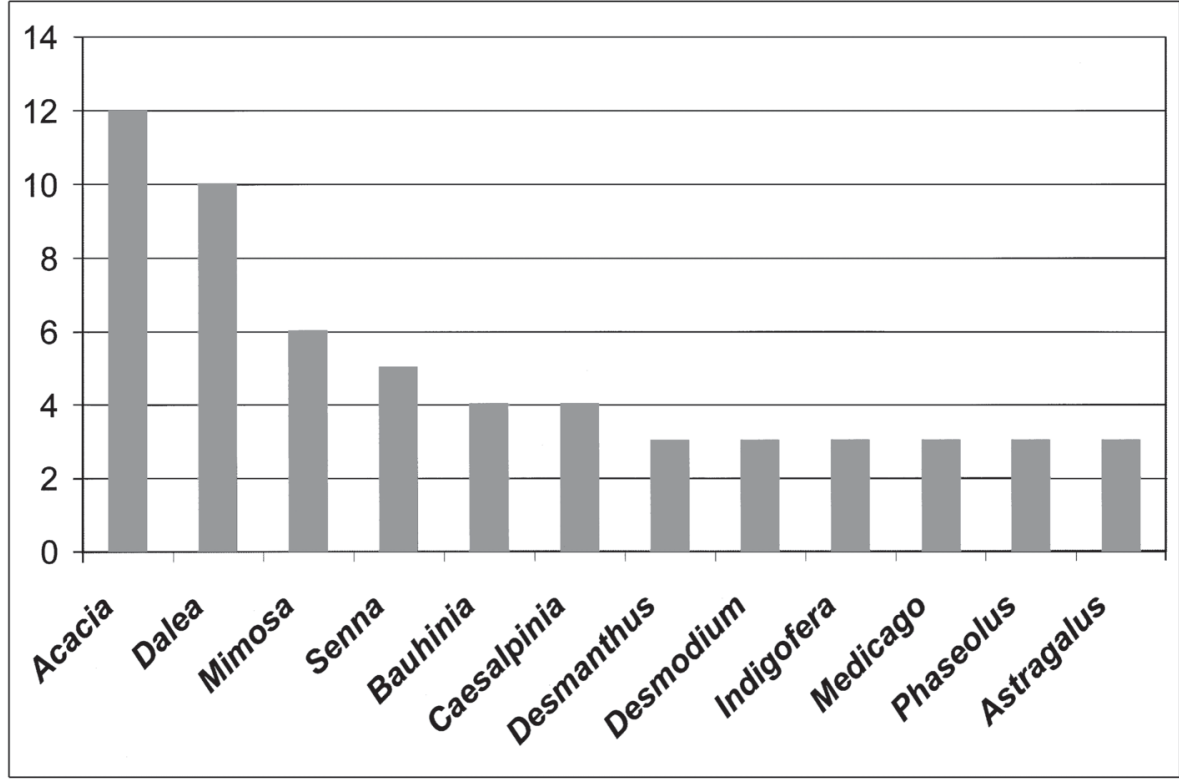

Fig. 3. Géneros con mayor número de especies. 
Las leguminosas arbustivas son las más abundantes en la zona, representan $47 \%$ (46 especies), de las cuales 23 son espinosas y 23 inermes, las herbáceas constituyen $46 \%$ ( 45 especies), mientras que las arbóreas integran $7 \%$ (7 especies). La mayor cantidad de taxa se registró en el matorral subinerme (52), matorral submontano (48), bosque de encino-pino (44) y, a su vez, los mezquitales albergan el menor número (23). Las Mimosaceae están más diversificadas en el matorral subinerme (21 especies) y matorral submontano (19 especies), mientras que en cada una de las dos comunidades restantes prosperan únicamente 13. Las Caesalpiniaceae son las menos numerosas de las tres familias, pues están representadas por 14 especies en el matorral subinerme, 5 en el matorral submontano, 5 en el bosque de encino-pino y 4 en el mezquital. Las Fabaceae son más abundantes en el bosque de encino-pino (26 especies) y matorral submontano (24), su diversidad disminuye a 17 especies en el matorral subinerme y sólo a 6 en las áreas de mezquital.

Seis especies restringen su distribución al noreste de México: Desmanthus velutinus, Ebenopsis ebano, Leucaena greggii, Bauhinia lunarioides, Astragalus greggii y Myrospermum sousanum. A su vez otras seis, Dalea hospes, Chamaecrista greggii, Cercidium macrum, C. texanum, Lupinus texensis y Gleditsia triacanthos extienden su distribución desde el noreste de México hasta el sur de los Estados Unidos. Cinco especies se cultivan como ornamentales: Delonix regia, Senna alata, Bauhinia purpurea, Caesalpinia gilliesii y C. pulcherrima. Una se siembra como forraje para ganado (Medicago sativa) y otra como alimento humano (Phaseolus vulgaris).

En la Sierra de Lampazos, municipio de Villaldama, se encontró la segunda población silvestre conocida para México de Gleditsia triacanthos ("árbol de la cruz"') asociada a comunidades de encino entre los 1200 y los 1300 m de altitud (Estrada et al., 2002). Esta especie se conocía previamente sólo de la Sierra de San Carlos, Tamaulipas (Briones, 1988). La población consta de 22 individuos y el área que ocupa no excede 1 ha. En la misma zona se documentó asimismo la presencia de una población de 30 individuos de Myrospermum sousanum, elemento para el cual sólo se tenían registros de la Sierra de Bustamante, municipio de Bustamante; Sierra de Gomas, municipio de Villaldama y Ojo de Agua de Agualeguas, en el municipio de Agualeguas (Delgado y Johnston, 1984).

Todas las especies de Acacia, Desmanthus, Mimosa y Prosopis habitan en las comunidades de matorral.

Acacia es el género con mayor presencia en la vegetación del norte de Nuevo León, pues sus especies se distribuyen en toda el área, sobresaliendo en términos de cobertura y densidad en las planicies. En especial, constituyen pieza esencial de las comunidades Acacia rigidula, A. neovernicosa y A. berlandieri, que definen parte del matorral subinerme y donde también es frecuente encontrar a Havardia pallens, Eysenhardtia texana y Calliandra conferta. 
Acacia farnesiana y Leucaena leucocephala se observan en toda la superficie estudiada, la primera se encuentra en casi todas las zonas que han sido sujetas a manejo mediante pastoreo o cultivos y posteriormente abandonadas, acompañada generalmente por Mimosa aculeaticarpa var. biuncifera, mientras que Leucaena leucocephala se observa en sitios invariablemente cercanos a asentamientos humanos (en patios de casas, a orillas de caminos, o bien como planta cultivada).

Con excepción del taxon cultivado, las especies de Bauhinia se distribuyen en el matorral submontano y bosque de encino. La más conspicua es $B$. macranthera por su porte de $3-5 \mathrm{~m}$ de altura. B. lunarioides y $B$. ramosissima son plantas arbustivas que regularmente no sobrepasan la talla de $2 \mathrm{~m}$ de altura y son menos frecuentes.

Se registraron cinco taxa con distribución restringida dentro del área de estudio, Acacia angustissima var. leucothrix y A. schaffneri var. bravoensis limitan su distribución al extremo noroeste del estado, en suelos arenosos, donde predominan las comunidades de mezquital. Cercidium texanum sólo se registró en las planicies en los municipios del extremo norte de Nuevo León en matorrales subinermes y mezquitales. Prosopis reptans var. cinerascens crece en áreas de planicie en suelos con alto contenido de sales, donde la vegetación es escasa, con claro predominio de Atriplex canescens, Suaeda nigrescens y Lycium berlandieri. Astragalus greggii se registró sólo en las montañas de Bustamante y Villaldama en las mismas áreas donde existe Myrospermum. Con anterioridad únicamente se tenían registros de este taxon para las montañas ubicadas en la frontera geopolítica de Coahuila y Nuevo León y el municipio de Múzquiz (Coah.) (Barneby, 1964).

Las especies de Dalea así como Rhynchosia senna var. angustifolia se distribuyen en todas las comunidades vegetales. Diphysa microphylla y D. occidentalis sólo existen en el matorral subinerme con apariciones discretas.

\section{DISCUSIÓN Y CONCLUSIONES}

Las condiciones de relieve, clima y suelo juegan un papel importante en la distribución de los diferentes taxa. La presencia de un mayor número de géneros y especies de la familia Mimosaceae en planicies y lomeríos bajos corresponde a la distribución global de la familia. De acuerdo con Barneby (1989) y Elias (1974), sus representantes prevalecen en los bosques ecuatoriales, bosques tropicales, sabanas y desiertos, con pocos elementos que penetran en áreas templadas.

Todos los géneros de Mimosaceae registrados del norte del estado de Nuevo León se distribuyen en la región norte de Tamaulipas. De manera similar, todas las especies, con excepción de Leucaena greggii son compartidas por ambas regiones. La afinidad climática y de relieve del norte de Nuevo León y Tamaulipas 
con la región del extremo sur de Texas es tal que, de las 30 especies registradas de Mimosaceae en el área de estudio, 26 se localizan en las planicies y lomeríos del sur de Texas, faltando sólo Acacia coulteri, A. parviflora, Desmanthus painteri, Leucaena greggii y Painteria elachistophylla.

La familia Caesalpiniaceae es más diversa y abundante en bosques ecuatoriales húmedos y sabanas con pocos miembros xerofíticos en desiertos cálidos (Robertson y Lee, 1976; Barneby, 1989), por lo que la escasa presencia de géneros y especies de este grupo de plantas se relaciona con las condiciones semiáridas que prevalecen en el norte de Nuevo León. Los taxa más abundantes y mejor representados en el matorral subinerme (sin alcanzar dominancia sobre las demás especies) son Cercidium macrum, C. texanum y Parkinsonia aculeata. Cinco especies cultivadas para ornato pertenecen a esta familia, todas ellas observadas en las planicies, donde las temperaturas bajas no son tan extremas como en las áreas de las zonas montañosas.

Los registros actuales que se tienen sugieren que Gleditsia triacanthos es rara en el norte de México, pues crece únicamente en las Sierras de San Carlos, Tamaulipas y de Lampazos, Nuevo León, en pequeñas poblaciones disyuntas, separadas por aproximadamente $270 \mathrm{~km}$. Tal discontinuidad de su distribución puede ser interpretada como fragmentos relictuales de una antigua área ininterrumpida. Las condiciones prevalecientes de clima, suelo y fisiografía de esta área son ligera o enteramente diferentes de las encontradas en varios sectores de los Estados Unidos en los que crece Gleditsia de donde esta planta se registra, a mencionar: suelos arcillosos y pendientes pedregosas (Stubbendiek y Elverne, 1989), como tolerante a suelos ácidos (Vines, 1960), creciendo entre 610-1500 m de altura (Spencer, 1947), como resistente a la sequía (Townsend, 1989), presente en bosques abiertos (Duncan y Duncan, 1988), como componente menor de las comunidades de bosque donde prospera (Blair, 1990) e intolerante a la sombra (Boyce, 1938).

La familia Fabaceae es de distribución cosmopolita, está más diversificada en las selvas tropicales cálidas secas, en áreas con clima templado y en las sabanas (Barneby, 1989). Esta preferencia de las especies por hábitats con climas más frescos en las montañas es característica de varios géneros en el norte de México y sur de los Estados Unidos, destacan entre ellos Canavalia, Lupinus, Desmodium, Phaseolus (silvestres), Calia y Vicia, la mayoría de cuyas especies concentran su distribución en las zonas menos cálidas.

De acuerdo con los criterios establecidos por la Unión Internacional para la Conservación de la Naturaleza en cuanto a especies amenazadas (Walter y Gillett, 1997), Gleditsia triacanthos y Myrospermum sousanum deben quedar incluidas dentro de la categoría de vulnerables. Las poblaciones de ambos taxa en México presentan una distribución restringida, están severamente fragmentadas y no existen en más de 10 localidades. 


\section{AGRADECIMIENTOS}

Deseamos agradecer al Consejo Nacional de Ciencia y Tecnología la ayuda económica brindada (Proyecto 34033-V, "Leguminosas del estado de Nuevo León") y al Programa de Apoyo a la Investigación Científica y Tecnológica de la Universidad Autónoma de Nuevo León por la infraestructura necesaria y apoyo económico para el estudio. Además, debemos el reconocimiento a Rosa María Murillo, a los revisores anónimos de esta revista y a Miguel Á. González B., quien elaboró el mapa y los cuadros.

\section{LITERATURA CITADA}

Anónimo. 1981. Síntesis geográfica del estado de Nuevo León. Instituto Nacional de Estadística Geografía e Informática. México D.F. 170 pp. + 13 mapas.

Barneby, R. C. 1964. Atlas of North American Astragalus. Part I-II, Mem. New York Botanical Garden 13:1-1188.

Barneby, R. C. 1989. Fabales In: Intermountain flora. Fabales. Vol. 3 parte B. New York Botanical Garden. Bronx, Nueva York. 279 pp..

Blair, R. 1990. Gleditsia triacanthos L. honeylocust In: Silvics of North America. Vol. 2: Hardwoods. Agricultural Handbook 654. United States Department of Agriculture, Forest Service. Washington, D.C. p. 211.

Boyce, J. 1938. Forest pathology. McGraw-Hill. Nueva York. 123 pp.

Briones, O. L. 1988. Nuevo registro para México de Gleditsia (Leguminosae). Bol. Soc. Bot. Mex. 48:143-144.

Cronquist, A. 1988. The evolution and classification of flowering plants. New York Botanical Garden. Bronx, Nueva York. pp. 371-373.

Delgado, A. y M. C. Johnston. 1984. A new species of Myrospermum (LeguminosaePapilionoideae) from northeastern Mexico. Syst. Bot. 9: 356-358.

Duncan, W. y H. Duncan. 1988. Trees of the southeastern United States. University of Georgia Press. Athens. 118 pp.

Elias, T. S. 1974. The genera of Mimosoideae (Leguminosae) in the southern United States. J. Arnold Arbor. 55: 67-113.

Estrada, A. E. y A. Martínez. 2000. Legumes from the central part of the state of Chihuahua, México. Sida 19(2): 351-360.

Estrada, A. E., E. Jurado y J. Jiménez. 2002. New locality of Gleditsia triacanthos (Caesalpiniaceae) in northeastern Mexico and its phytogeographic interest. Southwestern Naturalist 47(4): 602-604.

García, E. 1973. Modificaciones al sistema de clasificación climática de Köppen. 2a. ed. Instituto de Geografía. Universidad Nacional Autónoma de México. México, D.F. $217 \mathrm{pp}$.

Johnston, M. C. 1963. Past and present grasslands of southern Texas and northeastern Mexico. Ecology 44: 456-466. 
Lewis, G. P. 1998. Caesalpinia, a revision of the Poincianella-Erythrostemon group. Royal Botanic Gardens. Kew. 233 pp.

Miranda, F. y E. Hernández X. 1963. Los tipos de vegetación de México y su clasificación. Bol. Soc. Bot. Mex. 28: 29-179.

Muller, C. H. 1939. Relation of the vegetation and climatic types in Nuevo Leon, México. Amer. Midl. Nat. 21: 687-729.

Robertson, K. R. y Yin-tse Lee. 1976. The genera of Caesalpinioideae (Leguminosae) in the southeastern United States. J. Arnold Arbor. 57:1-53.

Rojas-Mendoza, P. 1965. Generalidades sobre la vegetación del estado de Nuevo León y datos acerca de su flora. Tesis de doctorado. Facultad de Ciencias, Universidad Nacional Autónoma de México. México, D.F. 124 pp.

Rzedowski, J. 1978. Vegetación de México. Ed. Limusa. México. D.F. pp. 97-110.

Spencer, C. 1947. Propagation of thornless honeylocust. Jour. Forest. 45: 715-722.

Stubbendiek, J., y C. Elverne. 1989. Common legumes of the Great Plains: an illustrated guide. University of Nebraska Press. Lincoln. 112 pp.

Townsend, A. M. 1989. The search for salt tolerant trees. Arbor. Jour. 13: 67-73.

Vines, R. A. 1960. Trees, shrubs and woody vines of the Southwest. Universisty of Texas Press. Austin. 1104 pp.

Walter, K. S. and Gillett, H. J. (eds.). 1998. 1997 IUCN Red list of threatened plants. Compiled by the World Conservation Monitoring Centre. IUCN - The World Conservation Union, Gland, Switzerland and Cambridge, UK. lxiv +862 pp.

White, S. S. 1940. Vegetation of the Cerro de la Silla, near Monterrey, Mexico. Mich. Acad. Sci. Papers 46: 87-98. 
Apéndice. Lista de especies de leguminosas registradas para el norte del estado de Nuevo León. Los números representan el número de colecta del primer autor. Comunidades vegetales en las que prosperan: EP (bosque de encino-pino), MSI (matorral subinerme), MSU (matorral submontano), MZ (mezquital). FLO (floración, mes(es)), IA (intervalo altitudinal).

\section{MIMOSACEAE}

Acacia angustissima (Mill.) Kuntze var. angustissima, 12385 EP, MSU. FLO, julio- agosto. IA, $700-950$ m s.n.m.

Acacia angustissima (Mill.) Kuntze var. leucothrix (Standl.) B.L. Turner, 12545 MZ. FLO, agosto-septiembre. IA, 600-850 m s.n.m.

Acacia berlandieri Benth., 11845; 11915; 11939a; 11997; 12129; 12184; 12201; 12409; $12416 ; 12465 ; 12849 ; 12918 ; 12972$ EP, MSU, MZ. FLO, febrero-julio. IA, 3701300 m s.n.m.

Acacia constricta Benth., 12785a MSI. FLO, marzo-julio. IA, 400-670 m s.n.m.

Acacia coulteri Benth., 12896 EP, MSU. FLO, abril-junio. IA, 750-950 m s.n.m..

Acacia farnesiana (L.) Willd., 11866; 12130; 12784a; 12910; 13017; 13065 EP, MSI, MSU, MZ. FLO, febrero-julio. IA, 350-1300 m s.n.m.

Acacia greggii A. Gray var. greggii, 11848; 12121; 12480; 12500; 12765b; 12783a; 12794c; 12852; 12892; 12893; 13027 MSI. FLO, Abril-Agosto. IA, 350-500 m s.n.m.

Acacia neovernicosa Isely, 12122; 12123; 12990; 13043 MSI. FLO, abril-agosto. IA, 400650 m s.n.m.

Acacia parviflora Little, 12014 MSI, MSU. FLO, abril-mayo. IA, 560-780 m s.n.m.

Acacia rigidula Benth., 11846; 12077; 12198; 12412; 12467; 12471; 12765a; 12785d MSI, MSU, MZ. FLO, febrero-abril. IA, 350-1200 m s.n.m.

Acacia roemeriana Scheele, 12074; 12172; 12196; 12917 EP, MSI. MSU. FLO, junio-julio. IA, 450-900 m s.n.m.

Acacia schaffneri (S. Watson) F. S. Herm. var. bravoensis Isely, 11851; 11877; 11925; 11958 MZ. FLO, marzo. IA, 290-430 m s.n.m..

Acacia schaffneri (S. Watson) F. S. Herm. var. schaffneri, 12856 MSI, MZ. FLO, marzo. IA, 340- 450 m s.n.m. 
Apéndice. Continuación.

Acacia wrightii Benth. ex A. Gray, 12420; 12433; 12783d; 13016; 13024 MSI, MZ. FLO, abril-agosto. IA, 330-460 m s.n.m.

Calliandra conferta Benth., 11827; 11964; 12202; 12236; 12362; 12411; 12436; 12466; 12862; 12886; 12919 MSI, MSU, MZ. FLO, abril-agosto. IA, 290-650 m s.n.m.

Desmanthus painteri (Britton \& Rose) Standl., 12438 MZ. FLO, abril. IA, 300- 870 m s.n.m.

Desmanthus velutinus Scheele, 12352 MSI. FLO, mayo-junio. IA, 380-580 m s.n.m.

Desmanthus virgatus (L.) Willd., 11869; 12454; 12473; 12488; 12492; 12800; 12828; 12848; 12866; 12895; 12907 EP, MSI, MSU, MZ. FLO, abril-septiembre. IA, 2901200 m s.n.m.

Ebenopsis ebano (Berland.) Barneby \& J. W. Grimes, 12117; 12492; 12884; 12890; 12909 MSI. FLO, febrero-marzo. IA, 320-450 m s.n.m.

Havardia pallens (Benth.) Britton \& Rose, 12227; 12796; 12850; 12889; 13060 MSI, MSU. FLO, mayo-julio. IA, 320-980 m s.n.m.

Leucaena greggii S. Watson, 11980; 12051; 12216; 12930; 12933 EP, MSU. FLO, abriljulio. IA, 630-1300 m s.n.m.

Leucaena leucocephala (Lam.) deWit. ssp. glabrata (Rose) S. Zárate, 11920; 12498; 12883 MSI, MZ. FLO, abril-junio. IA, 300-650 m s.n.m.

Mimosa aculeaticarpa Gómez-Ortega var. biuncifera (Benth.) Barneby, 12199; 13066 EP, MSU. FLO, IA, mayo-junio. 850-1280 m s.n.m.

Mimosa emoryana Benth. var. emoryana, 12992; 13031 MSI. FLO, IA, junio-agosto. IA, 380-660 m s.n.m.

Mimosa malacophylla A. Gray, 11993; 12186; 12802; 12810; 12888; 12908 MSI, MSU. FLO, abril-septiembre. IA, 400-840 m s.n.m.

Mimosa quadrivalvis L. var. laetidens (Small) Barneby, 12723 EP, MSU. FLO, abril-mayo. IA, $670-1260$ m s.n.m.

Mimosa texana (A. Gray) Small, 13046; 13064; EP, MSU. FLO, abril-mayo, agostoseptiembre. IA, 710-1280 m s.n.m. 
Apéndice. Continuación.

Mimosa zygophylla Benth., 12785b; 12794b; 13067; 13068; 13071 MSI. FLO, mayo-junio. IA, 400-560 m s.n.m.

Painteria elachistophylla (S. Watson) Britton \& Rose, 13054 EP, MSU. FLO, marzo-abril. IA, 670-1350 m s.n.m.

Prosopis glandulosa Torr. var. glandulosa, 11939; 12120; 12418; 12484; 12794d; 12894 MSI, MSU, MZ. FLO, marzo-abril. IA, 320-780 m s.n.m.

Prosopis glandulosa Torr. var. torreyana (L. Benson) M. C. Johnst., 11838; 11841; 12428; $12507 ; 12765 \mathrm{c} ; 12783 \mathrm{c} ; 12798 ; 12851 ; 12861 ; 13063$ EP, MSI, MSU, MZ. FLO, fabrero-abril. IA, 350-1250 m s.n.m.

Prosopis reptans Benth var. cinerascens (A. Gray) Burkart, 12124; 12783b MSI. FLO, mayo-junio. IA, 320-450 m s.n.m..

Zapoteca media (M. Martens \& Galeotti) H. M. Hern, EP, MSU. FLO, abril-mayo. IA, 6801230 m s.n.m.

\section{CAESALPINIACEAE}

Bauhinia lunarioides A. Gray ex S. Watson, 11987; 12006; 12035; 12073; 12363; 12825; 12859; 12931; 12973 EP, MSI. FLO, abril. IA, 740-1320 m s.n.m.

Bauhinia macranthera Benth. ex Hemsl., 12159; 13004 EP, MSU. FLO, abril-junio. IA, 750-1270 m s.n.m.

Bauhinia ramosissima Benth. ex Hemsl., 12879 EP, MSU. FLO, mayo-junio. IA, 670-1350 m s.n.m.

Bauhinia purpurea L., 1313, cultivada. FLO, marzo-mayo. IA, 290-370 m s.n.m..

Caesalpinia caudata (A. Gray) E.M. Fisher, (26 mi E of General Bravo, 27-XI-1966, Ripley \& Barneby 14783a (NY), Lewis, 1998). MSI. FLO, julio. IA, 200-380 m s.n.m.

Caesalpinia gilliesii (Wal. ex Hook.) Dietr., 12682, cultivada. FLO, mayo-julio. IA, 360620 m s.n.m.

Caesalpinia mexicana A. Gray, 12846; 12860 MSI, MSU. FLO, IA, mayo-julio. 360-850 m s.n.m. 
Apéndice. Continuación.

Caesalpinia pulcherrima L., 12306, cultivada. FLO, marzo-mayo. IA, 290-450 m s.n.m.

Cercidium macrum I. M. Johnst., 12422; 12425; 12427; 12435; 12448; 12452; 12478; 12491; 12855; 12887 MSI, MZ. FLO, abril-junio. IA, 280-470 m s.n.m.

Cercidium texanum A. Gray, 11852; 11956; 12119; 12496; 12506; 12784b; 12854; 12988 MSI, MZ. FLO, mayo-julio. IA, 300-450 m s.n.m.

Chamaecrista greggii (A. Gray) Pollard ex A. Heller var. greggii, 11959; 12036; 12071; $12348 ; 12413 ; 12444 ; 12459 ; 12470 ; 12503 ; 12505 ; 12801 ; 12827 ; 12847 ; 12863$; 12989 EP, MSI, MSU, MZ. FLO, abril-junio. IA, 290-900 m s.n.m.

Delonix regia (Boj. ex Hook.) Raf., 12309 cultivado. FLO, abril-julio. IA, 290-560 m s.n.m.

Gleditsia triacanthos L., 12378 EP. FLO, abril. IA, 1200-1300 m s.n.m.

Hoffmanseggia glauca (Gómez-Ortega) Eifert, 12945 MSI. FLO, marzo-agosto. IA, 350570 m s.n.m.

Hoffmanseggia oxycarpa Benth. ex A. Gray ssp. oxycarpa, 12218; 12224; 12464; 12932 MSI. FLO, abril-agosto. IA, 350-560 m s.n.m..

Parkinsonia aculeata L., 11949a; 12489; 12499 MSI. FLO, marzo-mayo. IA, 360-580 m s.n.m.

Pomaria canescens (Fisher) B. B. Simpson, MSI. FLO, mayo-agosto. IA, 290-610 m s.n.m.

Senna alata (L.) Roxb., 1222, cultivada. FLO, mayo-junio. IA, 290-530 m s.n.m.

Senna bauhinioides (A. Gray) H. S. Irwin \& Barneby, 11966; 12457; 12797; 12853; 12864 MSI. FLO, marzo-septiembre. IA, 320-480 m s.n.m.

Senna durangensis (Rose) H.S Irwin \& Barneby, 12469; 13048, MSI. FLO, octubre. IA, 310-510 m s.n.m.

Senna lindheimeriana (Scheele) H. S. Irwin \& Barneby, 12000; 12477 EP, MSI, MSU, MZ. FLO, julio-septiembre. IA, 350-870 m s.n.m.

Senna roemeriana (Scheele) H. S. Irwin \& Barneby, 13121. MSI. FLO, julio-agosto, 600750 m s.n.m. 
Apéndice. Continuación.

\section{FABACEAE}

Astragalus emoryanus Rydb. var. emoryanus, 15134, MSI, FLO abril-junio. IA, 120-560 m s.n.m.

Astragalus greggii S. Watson, 12002; 12136; 12356; 12376 EP. FLO, abril. IA, 1100-1300 m s.n.m.

Astragalus nuttallianus (DC.) var. austrinus (Small) Barneby, 11899 MSI, MZ. FLO, abriljunio. IA, 320-480 m s.n.m.

Calia secundiflora (Gómez-Ortega) Yakovlev, 12048; 12055; 12219; 12404; 12954 EP, MSU. FLO, marzo-abril. IA, 710-1280 m s.n.m..

Canavalia villosa Benth., 12980 EP. FLO, septiembre-octubre. IA, 900-1400 m s.n.m.

Dalea frutescens A. Gray, 12799 MSI, MSU. FLO, junio. IA, 380-750 m s.n.m.

Dalea greggii A. Gray, 12807 EP, MSI, MSU. FLO, febrero-abril. IA, 430-980 m s.n.m.

Dalea hospes (Rose) Bullock, 12395; 12494; 12953 EP, MSU. FLO, mayo-julio. IA, 7601300 m s.n.m.

Dalea laniceps Barneby, 12019; 12213; 12440 MSI, MZ. FLO, abril. IA, 300-490 m s.n.m.

Dalea lutea (Cav.) Willd. var. lutea, 12146; 12344; 12386; 12394 EP, MSU. FLO, marzoabril, octubre-noviembre. IA, 720-1320 m s.n.m.

Dalea nana Torr. var. carnescens (Rydb.) Kearney \& Peebles, 11872; 11909; 12017; 12025; $12111 ; 12115 ; 12200 ; 12410 ; 12419 ; 12439 ; 12460 ; 12891 ; 12987$ MSI, MZ. FLO, marzo-mayo. IA, 300-450 m s.n.m.

Dalea neomexicana (A. Gray) Cory var. longipila (Rydb.) Barneby, 11868; 12502; 12504; 12857; 12865; 12865 MSI, MZ. FLO, abril-julio. IA, 310-460 m s.n.m.

Dalea parrasana Brandegee, T. F. Patterson 6525 (TEX). MSU.

Dalea pogonathera A. Gray var. pogonathera, Wilson 10810 (TEX); Lavin 4512 (TEX). MSI. 
Apéndice. Continuación.

Dalea pogonathera A. Gray var. walkerae (Tharp \& Barckl.) B.L. Turner, 11858; 11904; $11932 ; 11943 ; 12113 ; 12212 ; 12423 ; 12442 ; 12460 ; 12479 ; 12794 a ; 12986 ; 13029$ MSI, MZ. FLO, abril-junio. IA, 290-530 m s.n.m.

Dalea wrightii A. Gray, T. F. Patterson 6815 (TEX); Hinton et al 21146 (TEX). MSI.

Desmodium lindheimeri Vail., 12187 EP, MSU. FLO, octubre. IA, 730-1280 m s.n.m.

Desmodium psilophyllum Schltdl., 12397 EP, MSU. FLO, agosto-octubre. IA, 740-1370 m s.n.m.

Desmodium retinens Schltdl., 12396 EP, MSU. FLO, marzo-mayo. IA, 820-1340 m s.n.m..

Diphysa microphylla Rydb., 13025 MSI. FLO, julio. IA, 350-540 m s.n.m.

Diphysa occidentalis Rose, 12430 MSI. FLO, junio-julio. IA, 320-490 m s.n.m.

Eysenhardtia texana Scheele, 11830; 11946; 11965; 12145; 12414; 12497; 12785c; 12826; $12858 ; 13002 ; 13028 ; 13030 ; 13045 ; 13053$ EP, MSI, MSU. FLO, abril-julio. IA, 330-1250 m s.n.m.

Galactia brachystachya Benth., 12038; 12079 EP, MSU. FLO, abril-mayo. IA, 750-1380 m s.n.m.

Galactia striata (Jacq.) Urban, EP, MSU. FLO, julio-agosto. IA, 700-1290 m s.n.m..

Indigofera lindheimeriana Scheele, 12110; 12371 EP, MSI, MSU. FLO, abril. IA, 310-1270 m s.n.m.

Indigofera miniata Gómez-Ortega var. miniata, 12078; 12112; 12434 EP, MSI, MSU. FLO, marzo-junio. IA, 340-1360 m s.n.m.

Indigofera suffruticosa Mill., 12044; 12509; 13015 EP, MSI, MSU. FLO, marzo-junio. IA, 290-1290 m s.n.m.

Lupinus texensis Hook., 12027; 12057 EP, MSU. FLO, abril-mayo. IA, 650-1100 m s.n.m.

Medicago lupulina L., 12523, EP, MSU. FLO, marzo-agosto. IA, 840-1370 m s.n.m.

Medicago polymorpha L., 12134 EP, MSU. FLO, abril-mayo. IA, 650-1650 m s.n.m. 
Apéndice. Continuación.

Medicago sativa L., 11916, cultivada. FLO, abril-junio. IA, 450-1300 m s.n.m..

Myrospermum sousanum A. Delgado \& M. C. Johnst., 12156; 12364; 12964; 13003 EP. FLO, mayo-junio. IA, 1200-1300 m s.n.m.

Nissolia platycalyx S. Watson, 11992; 12032; 12806 EP, MSU. FLO, mayo-junio. IA, 5901300 m s.n.m.

Nissolia platycarpa Benth., M. C. Johnston 2790 (TEX); Lavin 4515 (TEX); Villarreal 7500 (TEX); Villarreal 7475 (TEX). MSU.

Nissolia pringlei Rose, (2719, M. Carranza, 11-XII-1997, Sierra Pájaros Azules, Lampazos, N.L., TEX), EP. FLO, septiembre. MSU. IA, 990 m s.n.m.

Oxyrhynchus volubilis Brandeg. Villarreal 8035 (TEX). MSU.

Pediomelum rhombifolium (Torr. \& A. Gray) Rydb., 12391 EP, MSU. FLO, julio. IA, 6901350 m s.n.m.

Phaseolus maculatus (Scheele) ssp. ritensis (Jones) Frytag, 12147 EP;. FLO, junio-julio. IA, 1050-1380 m s.n.m.

Phaseolus neglectus Hermann, 12015; 12053 EP. FLO, octubre. IA, 980-1300 m s.n.m.

Phaseolus vulgaris L., 12056, cultivada. FLO, agosto-octubre. IA, 290-1200 m s.n.m.

Rhynchosia diversifolia M. Micheli var. prostrata Burkart, 12206; 12368; 12849a EP, MSI, MSU, MZ. FLO, abril-julio. IA, 320-1320 m s.n.m.

Vicia ludoviciana Nutt. ssp. ludoviciana, 11984; 12375; 12426 EP. FLO, junio-julio. IA, 970-1360 m s.n.m.

Vicia pulchella Kunth ssp. pulchella, 12398; 12490 EP. FLO, mayo-agosto. IA, 970-1480 m s.n.m. 